\section{Liver surgery: a long journey to improve results}

\author{
Isidoro Di Carlo*,1 \& Adriana Toro
}

\section{5th World Congress of the International Association of Surgeons, Gastroenterologists and Oncologists, Fuzhou, China, 4-6 September 2015}

Fuzhou, China hosted the 25th World Congress of the International Association of Surgeons, Gastroenterologists and Oncologists (IASGO). This was the first Congress after the passing away of Nicolas J Lygidakis, the founder of the International Association, who was a surgeon and an energetic secretary general of the association for 25 years. All members of the association are grateful for his message of medicine beyond the frontiers and health for all. The president of the association remains Professor Masatoshi Makuuchi, Emeritus Professor Of Surgery at The University of Tokyo (Japan), with Professor Dan Dunda from Harvard Medical School (MA, USA) and Professor Kyoichi Takaori from Kyoto University (Japan), as the secretaries general of the association. The President of the 2015 IASGO World Congress was Professor Yupei Zhao, Professor of Surgery at the University of Beijing and President of the Chinese Society of Surgery. The Congress was held under the auspices of IASGO president Masatoshi Makuuchi from Japan and both secretaries general. Fuzhou is a pleasant city that is well organized, but not as busy compared with other Chinese cities. All of the city's people were very kind and welcoming to the attendees at the meeting. The congress focuses on the major advancements in diagnosis and treatment of the gastroenterological diseases, here we report the most important progress in the field of hepatic surgery.

First draft submitted: 18 October 2015; Accepted for publication: 3 November 2015; Published online: 21 January 2016

Impact of serum CEA \& CA 19-9 levels for evaluation of recurrence \& survival in patients with colorectal liver metastases

Professor Toru Beppu from Kumamoto University (Japan), reported a very interesting and very large study on 1012 patients enrolled by the Japanese Society of Hepato-Biliary-Pancreatic Surgery. These patients had undergone hepatic resection for colorectal cancer. They had also received chemotherapy with oxaliplatin and irinotecan plus fluorouracil and leucovorin with or without molecular targeted drugs. The results of the study revealed that in a group of 727 patients with colorectal liver metastases treated by hepatectomy, a CA 19-9 level of more than $100 \mathrm{U} / \mathrm{ml}$ was considered to be an independent factor for both poor overall survival and recurrence, whereas CEA was not considered to be an independent factor with the same value. In a second group of 62 patients treated by hepatectomy

'Department of Surgical Sciences 'GF Ingrassia', University of Catania, Cannizzaro Hospital, Catania, Italy

${ }^{2}$ General Surgery, Patti Hospital, Messina, Italy

*Author for correspondence: Tel.: +39 095 726 4863; Fax: +39 095726 3020; idicarlo@unict.it

\section{KEYWORDS}

- hepatic arterial infusion chemotherapy - hepatocellular carcinoma - liver hemangiomas • liver metastases $\bullet$ TACE 
followed by chemotherapy, a high level of both markers after chemotherapy was considered to be an independent risk factor for both overall survival and recurrence. Therefore, the conclusion of the presentation by Professor Beppu was that both markers are useful in patients affected by colorectal cancer with liver metastases treated with different therapeutic modalities [1].

Sparing liver parenchyma to permit resection \& increase survival for patients with colorectal liver metastases \& hepatocellular carcinoma

Professor Hasegawa Kiyoshi from The University of Tokyo (Japan), presented two interesting videos concerning one of the most recent targets in hepatic surgery. In effect, surgical resection of both hepatocellular carcinoma (HCC) and colorectal liver metastases has been well established as the main method to cure patients with these diseases. At the present time, the liver parenchyma is spared in cases of HCC with insufficient liver function and in cases of colorectal liver metastases with a small future liver remnant. Currently, one more reason that needs to be applied to preservation of the liver parenchyma in both cases is the possibility of many reresections that can achieve long survival periods in these groups of patients. Of course, in some specific cases, the procedure can be very difficult and time consuming, as shown in the videos $[2,3]$.

How to achieve the best results in patients with unresectable HCC with marked arterioportal shunts using transarterial chemoembolization?

Professor Satoru Murata from Nippon Medical School presented a very interesting study on the treatment of patients affected by unresectable HCC with marked arterioportal shunts. Among 852 patients treated from 2005 to 2014 , 51 were diagnosed with arterioportal shunts. Of these patients, 24 received transarterial chemoembolization (TACE) plus portal vein occlusion (PVO) and 23 received TACE only including embolization of the arterioportal shunt. TACEPVO produced better results than TACE alone. The effectiveness of the first procedure was significantly higher $(\mathrm{p}<0.05)$, and the tumor response was significantly better in the first group $(\mathrm{p}<0.01)$, based on the median survival of 31 months in the first group and 16.8 months in the second group. This study by Professor Murata confirmed that TACE-PVO is superior to simple TACE alone in cases of HCC with arterioportal shunts [4].

\section{Back to arterial infusion for inoperable} HCC?

Patients with HCC at advanced stages have very few treatment options, and sorafenib is currently the most commonly used treatment. However, treatment with sorafenib is not very effective, and many complications are recorded. Hepatic arterial infusion chemotherapy has been considered useful in the past, because the first pass in the liver decreases the systemic side collateral effect, while simultaneously increasing the antitumor action. Professor Sung Suyun from Yeungnam University (South Korea), presented a study involving 46 patients affected by inoperable HCC. All of the patients were child class $\mathrm{A}$ and treated with neoadjuvant hepatic arterial infusion via a port inserted into the femoral artery. The doses of drugs used were $750 \mathrm{mg} / \mathrm{m}^{2}$ for 5 -FU and $25 \mathrm{mg} / \mathrm{m}^{2}$ for cisplatin. The patients were treated from day 1 to 4 , and the treatment was repeated for 4 weeks. The authors evaluated the tumoral responses using the Response Evaluation Criteria in Solid Tumors and the cumulative survival rates using the Kaplan-Meier method. A total of 20 patients were excluded from the study. The remaining 34 patients showed $11.8 \%$ complete response, $26.5 \%$ partial response, $47.1 \%$ stable disease and $14.7 \%$ progressive disease. The median survival was 10 months, and the 6-, 12-, 18- and 24-month cumulative survival rates were 87.5, $65.6,65.6$ and $65.6 \%$, respectively.

Eight patients underwent surgery, and had a median survival of 32.0 months. This relatively old technique was used massively [5] for liver metastases more than 10 years ago, can be considered current, and can be used to downstage the size of HCC to an operable state or used as a valid alternative in patients who remain inoperable [6].

\section{Giant liver hemangiomas: are they still giant?}

The term giant liver hemangioma is defined as a hemangioma larger than 4,5 or $10 \mathrm{~cm}$. The majority of studies reported in the literature have defined giant hemangiomas as those of more than $4 \mathrm{~cm}$. In addition, many reports in the literature have described surgical procedures based only on this terminology without any symptoms of the patients related to the 
tumors. My coworkers and I presented a new proposal for the definition of giant hemangioma to the Congress, based on both personal experience and data in the literature, describing that the term giant hemangioma should be used for tumors larger than $10 \mathrm{~cm}$. Conversely, tumors smaller than $5 \mathrm{~cm}$ are defined as small and need to undergo surgery in very exceptional cases, as follow-up of the patients is the medical behavior usually adopted for this size of tumor [7].

\section{References}

1 Sakamoto Y, Miyamoto Y, Beppu T et al. Post-chemotherapeutic CEA and CA19-9 are prognostic factors in patients with colorectal liver metastases treated with hepatic resection after oxaliplatin-based chemotherapy. Anticancer Res. 35, 2359-2368 (2015).

2 Viganò L, Capussotti L, Lapointe R et al. Early recurrence after liver resection for colorectal metastases: risk factors, prognosis, and treatment. A LiverMetSurvey-based study of 6,025 patients. Ann. Surg. Oncol. 21, 1276-1286 (2014).

\section{Financial \& competing interests disclosure}

The authors have no relevant affliations or financial involvement with any organization or entity with a financial interest in or financial conflict with the subject matter or materials discussed in the manuscript. This includes employment, consultancies, honoraria, stock ownership or options, expert testimony, grants or patents received or pending, or royalties.

No writing assistance was utilized in the production of this manuscript.

3 Chan AC, Poon RT, Chok KS et al. Feasibility of laparoscopic re-resection for patients with recurrent hepatocellular carcinoma. World J. Surg. 38, 1141-1146 (2014).

4 Chen J, Chen S, Xi W et al. Transcatheter arterial chemoembolization and chemotherapy plus sorafenib in a large hepatocellular carcinoma with arterioportal shunt. Case Rep. Oncol. Med.2014, 392403 (2014).

5 Seo JH, Song MJ. Complete remission after hepatic arterial infusion chemotherapy for advanced hepatocellular carcinoma with decompensated cirrhosis. J. Gastroenterol.
Hepatol. doi:10.1111/jgh.13033 (2015) (Epub ahead of print).

6 Kawaoka T, Aikata H, Hyogo $\mathrm{H}$ et al. Comparison of hepatic arterial infusion chemotherapy versus sorafenib monotherapy in patients with advanced hepatocellular carcinoma. J. Dig. Dis. 16, 505-512 (2015).

7 Di Carlo I, Koshy R, Ardiri A, Bertino G, Toro A. Giant cavernous liver hemangiomas - it is time to relook the size definition? New proposal based on last 40 years of the literature evidence. Hepatobiliary Pancreat. Dis. Int. doi:10.1016/ S1499-3872(15)60035-2 (2015) (Epub ahead of print). 